\title{
Application of the Dependency Graph Method in the Analysis of Automatic Transmission Gearboxes
}

\author{
Adam Deptuła \\ Department of Management and Production Engineering \\ Faculty of Production Engineering and Logistics \\ Opole University of Technology \\ Opole, Poland \\ a.deptula@po.edu.pl
}

\begin{abstract}
The current article discusses the use of the dependency graph method to design and analyze automatic transmissions. Different goals may be served by modeling an automatic transmission using graphs. The most important of them are: determining the gear ratios for gears and the analysis of speed and acceleration of the rotating elements. Game tree-structure methods can be used to analyze the functional schemes of the selected gears. This paper describes the method of generating a system of kinematics equations for signal dependency graphs. This allows the generalization and extension of the algorithmic approach, and also further analyses and syntheses, such as checking the isomorphism of the proposed solutions and determining the validity of the structure and the operational parameters of the analyzed gears.
\end{abstract}

Keywords-computational model; computer aided design; optimization; graph theory; mechanical engineering

\section{INTRODUCTION}

A machine as a system is a set of objects (blocks, elements), each of which is described by an appropriate mathematical model with indications of all connections existing between objects. System objects are usually individual devices: heat exchangers, extractors, compressors, etc. Nowadays, the use of artificial intelligence, expert systems, or neural networks is increasingly utilized to solve many technical and engineering problems [1]. Engineering practice requires a correct assessment of the mathematical model describing a given system by means of variables. Models describe a given system with different accuracy. Graphs and structural numbers play a role as models of mechanical systems [2-5] and are still systematically developed [6-11]. In addition, there are special stream graphs, e.g. in chemical and process engineering. Many optimization methods are used in technical solutions to improve the efficiency of internal combustion and electric vehicles $[12,13]$. Unlike graphs, dendrite-tree structures do not have cycles, but there may be a different number of initial vertices. Therefore, such structures are applicable to variant searching and optimizing the solutions of the designed system [6]. Some of the functional requirements are transformed into the structural characteristics and incorporated in the generator as rules of enumeration. The generator enumerates all possible solutions using graph theory and combinatorial analysis. The remaining functional requirements are incorporated in the evaluator as evaluation criteria for the selection of concepts [14]. This results in a class of feasible mechanisms. Finally, the most promising candidate is chosen for the product design.

The process may be iterated several times until the final product is achieved. This methodology has been successfully applied in the structure synthesis of epicyclic gear trains, automotive transmission mechanisms, variable-stroke engine mechanisms, robotic wrist mechanisms, etc. Generally, if a functional requirement can be written in a mathematical form, it should be included in the generator [2]. The current article presents the method of dependence graphs in the analysis of automatic transmission. The present work describes the algorithm of searching and generating parametric trees as outline graphs. The proposed method allows determining the optimal values of teeth, using only the relationships linking transmission elements with their presentation in the form of a graph. This allows a more accurate calculation of the number of teeth and an algorithmic approach to the problem. Methods using graphs and structural numbers have long been used in mechanics. Many previous works considered the theory of graphs as tools for studying system dynamics, both in the analysis and in the synthesis of complex mechanical systems. In particular, authors in $[15,16]$ focused on determining the power flow through closed kinematic chains. The circulating power dependency is used to analyze toothed gears. A graph describes the scalar product of the coupled graphs. The transmission system is described using kinematic chains, and the coupled toothed transmission system is described using block diagrams, which enables such systems to be analyzed in $n$ degrees. In addition, from the diagrams (blocks) describing the coupled transmission systems, the authors constructed coupled graphs and pseudo-graphs. By varying the associated values, optimization is performed to minimize the load on a given kinematic chain of the transmission system. This is a different way of using dependency graphs than that described in the present work. The present methodology is based on input and output information (values). The graphs described in the cited works are not subject to decomposition or distribution over decision trees. In seeking optimum decisions (paths) it is desirable to define the properties of the structure in such a way that the nodes represent the sought parameter, and the leaves 
determine the output decision that is optimum at a given moment, as a response of the system to a particular change. Decisions on the tree structure simulate a certain fictional "game" (mutual interaction) between the decision-maker (the decision taken) and the transmission system (the response to the decision).

\section{GRAPHIC TRANSMISSION MODELS}

The objectives of gear modeling with graphs vary: dynamic analysis, kinematic analysis [4], synthesis [17], structure analysis [18], enumeration [19], optimization of gear sequences, automatic design, etc. are based on the so-called graph grammars. The advantage of modeling gears with graphs is that issues considered using graph models can be solved in an algorithmic manner, which allows the use of computer programs and widely understood integrated decision-making systems in a simple manner. In graph theory, a graph is associated with many other algebraic structures as arrays, matroids, structural numbers, and linear spaces of cutoffs. These objects enable the coding of the transmission structure, which allows the use of advanced artificial intelligence (evolutionary, genetic, or immunological) algorithms. An important advantage of modeling mechanical systems with graphs is that some considerations can be carried out in parallel in the field of mechanics and graph theory [20]. The relevance of the results is based on the transformation of knowledge between these two areas. At present, there is a considerable interest in graphical methods in optimization, and especially in modeling of gears, hydraulic systems, mechanisms, trusses and frames. Among the methods of analysis of planetary gears, one can distinguish among others [5, 20-22]. In the case of Hsu rules, the graph is built according to the following rules: geometrical dimensions are omitted and the considered kinematic pairs are: rotary, "planet-yoke", and meshing. The contour graph method was discussed in $[6,7,22,23]$ for the analysis of mechanical systems. It is particularly useful for considering mechanisms of various types (so-called planar, crossheads, etc.). In particular, it can be used in the analysis of planetary gears. In the analysis of planetary gears, the contour graph method is used. This method is based on distinguishing a number of subsequent rigid links of mechanisms that form a closed loop, the so-called contour. In particular, [21] concerns only outline graphs and their applications.

Unlike graphs, dendrite-tree structures do not have cycles, but there may be a different number of initial vertices. Therefore, a different approach has been developed as a translation of a directed dependence graph, among others for game-tree structures. This approach is different from the previous literature on parametric automation machines and their applications, related to control systems, operating systems, importance analysis of construction and/or operating parameters, and gear analysis previously modeled using other graph types. Various methodologies have been developed for the systematic enumeration of kinematic structures. A thorough understanding of the structural characteristics of a given type of mechanism is critical for the development of an efficient algorithm. During the ' 80 s, some approaches for structural synthesis of kinematic chains were presented. Authors in [24] presented a systematic approach for the kinematic analysis of multi degree of freedom robotic bevel-gear trains. In the '00s, authors in $[25,26]$ presented the structure synthesis of mechanisms. Authors in [7] showed the kinematic, power flow, and efficiency analysis of epicyclic gear trains. Authors in [27] presented a survey of works connected with the problem of gear modeling by means of versatile graph theory models. More recently, authors in [28] studied the kinematic, static force, power flow, and efficiency analysis of gear trains. Other authors researched the topological synthesis of gear trains. The review analysis of the graph application issues in the transmission analysis can include kinematic analysis, torque analysis, power flow analysis, and mechanical efficiency analysis.

The general algorithm for gear modeling with graphs can be described as:

- Selecting a problem for kinematic analysis or synthesis and considering the elements related to it (abstracting).

- Determining the relationships for the specified elements (e.g. listing kinematic rotational pairs and meshing).

- Listing the selected subgraphs based on the basic transmission graph and saving the codes.

- Generating of the equations describing the gearing on the basis of codes - which is an orderly (algorithmic) way and solving the obtained system of equations.

\section{ANALYSIS OF THE AUTOMATIC TRANSMISSION GEARBOXES WITH GAME-TREE STRUCTURES}

\section{A. Automatic Transmission Gearboxes}

Modern automatic transmissions are complex devices, consisting of several hundred elements of a mechanicalhydraulic system and another several hundred in the electronic module. Automatic planetary gearboxes belong to a special type of gearbox. There are several of them, each of which consists of a sun gear with external meshing, satellites, and a crown gear with internal meshing. The specified gear ratio is implemented by braking or connecting a sun wheel or satellites. Brakes or clutches of individual planetary gears are controlled by hydraulic valves (transmission oil flow). The gearboxes are characterized by high durability and the ability to instantly change gears [29].

The current work is based on [6] concerning various possible developments of applications of graph methods in the analysis of gears. In particular, in [6] the concepts for describing the model of machine systems data using dependency graphs and signal flow were formulated. Among other things, the authors in [30] developed a method for finding the optimal number of teeth without transforming the graph. The algorithm for generating equations and determining the optimal number of teeth is calculated directly from the dependency graph considered as a signal flow graph. This approach is different from other works on the application of the graph method in gear analysis. For example, in [31], the gear analysis uses the Davie's equations and the interdependencies of cylindrical gear alignment. In turn, authors in [32] used hypergraphs to determine the relationship between elements and generate kinematic equations. The use of graph grammars 
for the description of graph chains in the description of the gearboxes was used in [28, 33]. This is a different approach from that used in the works discussed above. The construction of contours in a graph begins in the supporting system and passes through successive rotating gear elements, each of which transfers rotary motion to the next. The contour is closed when we return in the analysis to the supporting system. To create a complete graph, all contours must be analyzed. The main goal of kinematic analysis of a toothed gear is to determine the transmission ratio and to identify any surplus gears.

The analysis of automatic gearboxes is similar to the analysis of a single planetary gear. During the process of kinematic structure enumeration using graph theory, isomorphism identification of graphs is an important and complicated problem. There are many approaches to detect isomorphic graphs, and these approaches have been largely algorithmic. This path is formed by the corresponding edges of the gear graph. Input and output are marked additionally. This path allows the analysis of the sequence of transmission of rotational motion by the subsequent elements of the transmission. In addition, it allows the detection of the socalled redundant elements for the given gear which is currently under consideration. The main purpose of the kinematic analysis of the planetary gear is to determine the kinematic ratio and possibly detect oversized gears. One of the methods of analysis, based on the use of the Willis formula for any complex planetary gears, is described in detail in the [34], in which the theory of contour graphs was used for kinematic analysis. This method allowed not only to determine the kinematic ratio, i.e. the angular velocities of all wheels and gear yokes, but also to determine the angular accelerations of rotating gear elements. In addition, redundant (oversized) wheels could be detected when plotting contour graphs. Individual gears were implemented using brakes, clutches and backstops (free wheels) as shown in Table I [34].

TABLE I. FUNCTIONAL MATRIX OF THE A4LD GEARBOX

\begin{tabular}{|c|c|c|c|c|}
\hline Position & DE (1 2 3 4) & $\begin{array}{c}\mathbf{2}^{\text {nd }} \\
\text { gear }\end{array}$ & $\begin{array}{c}\mathbf{1}^{\text {th }} \\
\text { gear }\end{array}$ & Reverse \\
\hline MT cl & $(--\mathrm{X} \mathrm{X})$ & - & - & - \\
\hline cl1 & $(----)$ & $\mathrm{X}$ & $\mathrm{X}$ & $\mathrm{X}$ \\
\hline $\mathrm{b} 1$ & $(---\mathrm{X})$ & - & - & - \\
\hline Fw1 & $(\mathrm{X} \mathrm{XX} \mathrm{-)}$ & $\mathrm{X}$ & $\mathrm{X}$ & $\mathrm{X}$ \\
\hline $\mathrm{cl} 2$ & $(--\mathrm{X} X)$ & - & - & $\mathrm{X}$ \\
\hline $\mathrm{cl} 3$ & $(\mathrm{X} \mathrm{XXX)}$ & $\mathrm{X}$ & $\mathrm{X}$ & - \\
\hline b2 & $(-\mathrm{X}--)$ & $\mathrm{X}$ & - & - \\
\hline b3 & $(----)$ & - & $\mathrm{X}$ & $\mathrm{X}$ \\
\hline Fw2 & $(\mathrm{X}---)$ & - & - & - \\
\hline
\end{tabular}

The object of the analysis is a complex four-speed automatic transmission with overdrive type A4LD [29, 34].

\section{B. Dependency Graph and Game-Tree Structures}

A graph is an ordered pair $G=(V, E)$, in which $V$ is a fine set of elements called the vertices of the graph, and $E$ is a set of $\left(v_{i}, v_{j}\right)\left(v_{i}, v_{j} \in V\right)$ pairs called the edges of the graph. To fully specify the graph, one must also specify the relationship $P$ formed by the individual elements of the set of vertices $V(G)$ and edges $E(G)$. Then the graph can be called an ordered three:

$$
G=(V, E, P)
$$

where $V$ is the set of graph vertices, $E$ the set of edges (graph branches), and $P$ is the three-member relationship which meets the following condition: There is such a pair of vertices $x, y \in V$ for each branch $e$, such that $\langle x, e, y\rangle \in P$. If for branch $e$ exist $\langle x, e, y\rangle \in P$ and $\langle w, e, z\rangle \in P$ then either $x=w$ and $y=z$ or $x=y=z$. A dependency graph is an ordered pair $G=(X, R)$, in which $X$ is a finite set of elements called vertices of the graph, and $R$ is a set of pairs $\left(x_{i}, x_{j}\right)\left(x_{i}, x_{j} \in X\right)$ called the edges of the graph. In the case of parametric graphs, the notation introduced in [34] defines the signs: $G=(Q, Z)$, where $Z$ is a set of pairs $\left(z_{i}, z_{j}\right)\left(z_{i}, z_{j} \in Z\right)$. The oriented dependency (game) graph shown in Figure 1 is composed of a set of vertices $Q$ :

$$
Q=\left\{q_{1}, q_{2}, q_{3}, q_{4}, q_{5}\right\}
$$

and of set of edges Z:

$$
Z=\left\{z_{1}, z_{2}, z_{3}, z_{4}, z_{5}, z_{6}, z_{7}\right\}
$$

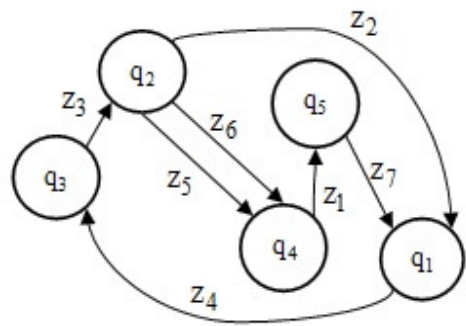

Fig. 1. An example of a dependency graph.

The path in the $G=(Q, Z)$ is the edge sequence $\left(z_{i_{1}}, z_{i_{2}}\right),\left(z_{i_{2}}, z_{i_{3}}\right), \ldots,\left(z_{i_{k-1}}, z_{i_{k}}\right)$ in which each $j \in\{2,3, \ldots, k\}\left(z_{i_{j-1}}, z_{i_{j}}\right) \in R$ and vertices $q_{i_{1}}, q_{i_{2}}, \ldots, q_{i_{k}}$ are different pairs. Vertex $q_{i_{1}}$ is called the beginning of the pat, and the top $q_{i_{k}}$ the end of the road. As a result of a graph distribution from the chosen vertex, a tree structure with cycles is obtained in the first step and then, a general game tree structure is obtained. Each of them has an appropriate analytical formulation $G_{i}^{+}$and $G_{i}^{++}$. A game tree structures is a part of the systematic searching method. The algorithm of graph distribution of dependence on parametric structures has been presented, among others, in [35]. The expression describing the degree of inferiority of a given component graph is marked with a pair of parentheses, inside which the expression concerning the given component graph is written. When starting to build an analytical expression representing a graph (Figure 1), first the starting vertex of the graph should be determined. In the case of starting the analysis from the initial vertex $q_{1}$, we get the following expressions: 


$$
\begin{gathered}
G(1)_{q 1}^{+}=\left({ } ^ { 0 } q _ { 1 } \left({ } ^ { 1 } z _ { 2 } q _ { 3 } { } ^ { 2 } { } ^ { 2 } z _ { 3 } q _ { 2 } \left({ }^{3} z_{4} q_{1}, z_{5} q_{4}\left({ }^{4} z_{1} q_{5}\left({ }^{5} z_{7} q_{1}\right)^{5}\right)^{4},\right.\right.\right. \\
\left.\left.\left.\left.z_{6} q_{4}\right)^{3}\right)^{2}\right)^{1}\right)^{0} \\
G(1)_{q 1}^{++}=\left({ } ^ { 0 } q _ { 1 } \left({ } ^ { 1 } z _ { 2 } q _ { 3 } \left({ } ^ { 2 } z _ { 3 } q _ { 2 } \left({ }^{3} z_{4} q_{1}^{1}, z_{5} q_{4}\left({ }^{4} z_{1} q_{5}\left({ }^{5} z_{7} q_{1}^{2}\right)^{5}\right)^{4},\right.\right.\right.\right. \\
\left.\left.\left.\left.z_{6} q_{4}\left({ }^{4} z_{1} q_{5}\left({ }^{5} z_{7} q_{1}^{3}\right)^{5}\right)^{4}\right)^{3}\right)^{2}\right)^{1}\right)^{0}
\end{gathered}
$$

\section{Kinematic ANALYSIS OF THE GEAR WITH THE USE OF} DEPENDENCY GRAPHS

Power is transmitted through the torque converter, $h_{1}$ yoke, free wheel $\mathrm{Fw}_{1}, \mathrm{Cl}_{3}$ and $\mathrm{Cl}_{2}$ clutches, gears 6, 5, 4 and yoke $h_{2}$ and in parallel through wheels $7,8,9$ on the output shaft II (thanks to the $F w_{2}$ backstop activated), as can be seen in Figure 2. The signal flow dependence graph of gears is shown in Figure 3 .

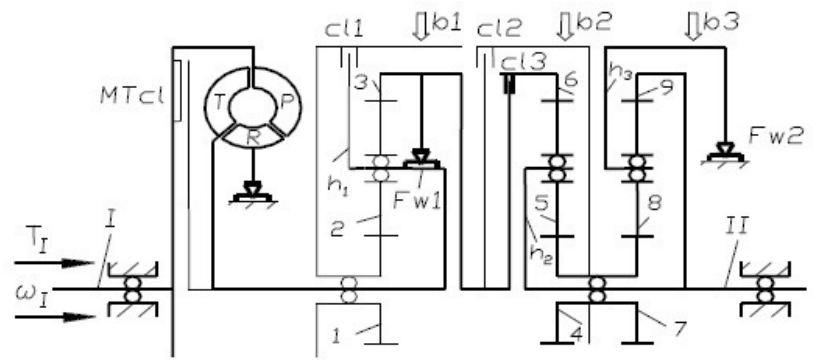

Fig. 2. Power efficiency diagram for DE1 gear.

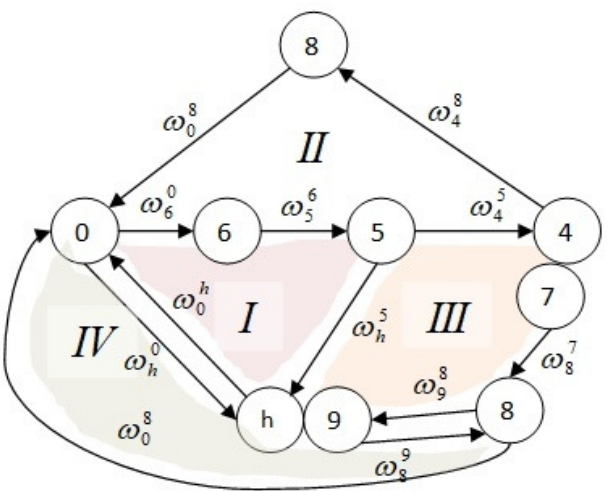

Fig. 3. Signal dependency graph for DE.

For the graph model, a system of contour equations of velocity $\omega_{i, i-1}$, peripheral velocities $\omega_{i, i-1} \times r_{A i}$, angular $\varepsilon_{i, i-1}$, tangential $\varepsilon_{i, i-1} \times r_{A i}$, and centripetal accelerations $\omega_{i}^{2} \cdot r_{A i, A i+1}$ was generated and can be seen in (6- 9):

$$
\left\{\begin{array}{l}
\sum_{(i)} \omega_{i, i-1}=0 \\
\omega_{6,0}+\omega_{5,6}+\omega_{h 2,5}+\omega_{0, h 2}=0 \\
\omega_{6,0}+\omega_{5,6}+\omega_{4,5}+\omega_{8,7}+\omega_{0,8}=0 \\
\omega_{4,5}+\omega_{8,7}+\omega_{9,8}+\omega_{5, h 2}=0 \\
\omega_{6,0}+\omega_{5,6}+\omega_{h 2,5}+\omega_{8,9}+\omega_{0,8}=0 \\
\sum_{(i)} r_{A i} \times \omega_{i, i-1}=0
\end{array}\right.
$$

$$
\left\{\begin{array}{l}
\sum_{(i)} r_{A i} \times \omega_{i, i-1}=0 \\
r_{6} \times \omega_{5,6}+\left(r_{4}+r_{5}\right) \times \omega_{h 2,5}=0 \\
r_{6} \times \omega_{5,6}+r_{4} \times \omega_{4,5}+r_{7} \times \omega_{8,7}+r_{9} \times \omega_{9,8}=0 \\
r_{4} \times \omega_{4,5}+r_{7} \times \omega_{8,7}+r_{9} \times \omega_{9,8}+\left(r_{4}+r_{5}\right) \times \omega_{5, h 2}=0 \\
r_{6} \times \omega_{5,6}+\left(r_{4}+r_{5}\right) \times \omega_{h 2,5}+r_{9} \times \omega_{8,9}=0
\end{array}\right.
$$

$$
\left\{\begin{array}{l}
\sum_{(i)} \varepsilon_{i, i-1}=0 \\
\varepsilon_{6,0}+\varepsilon_{5,6}+\varepsilon_{h 2,5}+\varepsilon_{0, h 2}=0 \\
\varepsilon_{6,0}+\varepsilon_{5,6}+\varepsilon_{4,5}+\varepsilon_{8,7}+\varepsilon_{0,8}=0 \\
\varepsilon_{4,5}+\varepsilon_{8,7}+\varepsilon_{9,8}+\varepsilon_{5, h 2}=0 \\
\varepsilon_{6,0}+\varepsilon_{5,6}+\varepsilon_{h 2,5}+\varepsilon_{8,9}+\varepsilon_{0,8}=0
\end{array}\right.
$$

$$
\left\{\begin{array}{l}
\sum_{(i)} r_{A i} \times \varepsilon_{i, j-1}-\omega_{i}^{2} \cdot r_{A i, A i+1}=0 \\
r_{6} \times \varepsilon_{5,6}+\left(r_{4}+r_{5}\right) \times \varepsilon_{h 2,5}=0 \\
r_{6} \times \varepsilon_{5,6}+r_{4} \times \varepsilon_{4,5}+r_{7} \times \varepsilon_{8,7}+r_{9} \times \varepsilon_{9,8}=0 \\
r_{4} \times \varepsilon_{4,5}+r_{7} \times \varepsilon_{8,7}+r_{9} \times \varepsilon_{9,8}+\left(r_{4}+r_{5}\right) \times \varepsilon_{5, h 2}=0 \\
i_{6,9}=\omega_{6,0} / \omega_{9,0}= \\
r_{6} \times \varepsilon_{5,6}+\left(r_{4}+r_{5}\right) \times \varepsilon_{h 2,5}+r_{9} \times \varepsilon_{8,9}=0
\end{array}\right.
$$

where $\omega_{i, i-1}$ is the relative angular velocity vector of the element $i$ relatively to the previous element $i-1, \omega_{i, 0}$ is the vector of the absolute angular velocity of the element (relative to the fixed base), $r_{A i}=r_{O A i}$ is the radius of the vector of point $A_{i}$ (point $A$ on element $i$ ), and $r_{A i, A i+1}=r_{A i+1}-r_{A i}, \varepsilon_{i, j-1}$ the vector of relative angular acceleration of the element relative to the previous element $i-1$.

\section{APPLICATION OF THE SIGNAL FLOW GRAPH METHOD IN CALCULATING THE NUMBER OF GEAR TEETH}

A new approach for the dependency graph can be obtained by using relationships that bind systems with their representation in the form of a dependency graph (in particular in relation to contour graphs). As mentioned above, a graph is an ordered pair $G=(V, E)$, in which $V$ represents its vertices and $E$ its edges. The attached $\widehat{G}$ search graph to a regular signal flow graph $G$ is defined by replacing all $G$ graph branches with the attached equivalents (Figure 4).

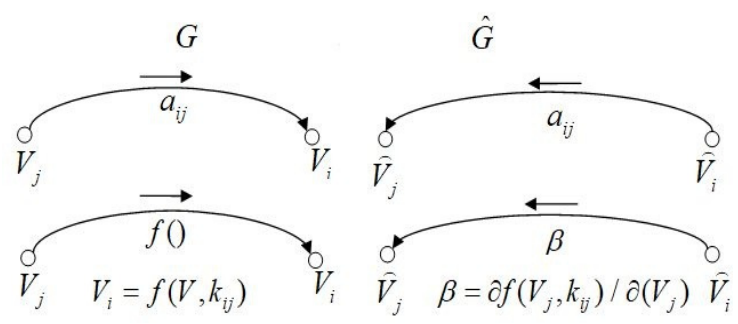

Fig. 4. Signal dependency graph for DE1. 
A search graph $\hat{G}$ for linear branches is created by reversing the direction of the signal flow. For the non-linear branch described by the function $f\left(V_{j}, k_{i j}\right)$ ( $k_{i j}$-number coefficient) in addition to reversing the direction of the branches, there is also a change in its description from $f\left(V_{j}, k_{i j}\right)$ to $\partial f\left(V_{j}, k_{i j}\right) / \partial\left(V_{j}\right)$, where the derivative is determined at the point $V_{j}$ corresponding to the solution of the graph $G$, i.e. searching the entire vertex $j$. This graph definition, which is the equivalent of the search circuit, allows to directly use the search graph method to calculate the sensitivity. The sensitivity of the $V_{o}$ output signal relative to the gain of the linear branch or nonlinear branch stick factor is determined on the basis of the solution of graphs $G$ and $\widehat{G}$ in the form of:

$$
\begin{aligned}
& \frac{\partial V_{O}}{\partial a_{i j}}=\hat{V}_{i} V_{j} \\
& \frac{\partial V_{O}}{\partial k_{i j}}=\hat{V}_{i} \frac{\partial f\left(V_{j}, k_{i j}\right)}{\partial k_{i j}}
\end{aligned}
$$

The sensitivity determination method applies to both coupled and unidirectional graphs. By minimally changing only the excitation value (e.g. the number of teeth) in the search graph $\widehat{G}$, the method can be adapted to determine the full gradient vector of the objective function [35]. The objective function will be expressed as the sum of squares of differences between the current values of the output signals $V_{o i}$ and the set values $d_{i}$ :

$$
E(W)=\frac{1}{2} \sum_{i=1}^{M}\left(V_{o i}-d_{i}\right)^{2}
$$

where:

$$
W=\left[W_{1}, W_{2}, \ldots, W_{N}\right]^{T}
$$

The gradient $\nabla E$ is obtained as:

$$
\nabla E=\left|\begin{array}{c}
\frac{\partial E}{\partial W_{1}} \\
\frac{\partial E}{\partial W_{2}} \\
\cdots \\
\frac{\partial E}{\partial W_{N}}
\end{array}\right|
$$

where:

$$
\frac{\partial E}{\partial W_{j}}=\sum_{i=1}^{M}\left(V_{o i}-d_{i}\right) \frac{\partial V_{o i}}{\partial W_{j}}
$$

The calculation of the $j$-th vector component of the gradient consists in the calculation of the derivative output signals $V_{o i}$ and multiplying them by the appropriate difference: $V_{o i}-d i$. The back propagation algorithm has been developed for use in unidirectional networks. Its interpretation and generalization in the form of graphs applies to both directed graphs and recursive networks containing feedback cycles, e.g. a drawing with trees. It should be noted that in the case of unidirectional networks, it is easy to extract successive layers of neurons and search the signal flow between them, while in the dependence graphs this task is more complicated due to the existing cycles (feedback and contours). Figure 5 shows an example of the implementation of the search graph. Figure 6 shows the signal response for the selected graph vertices.

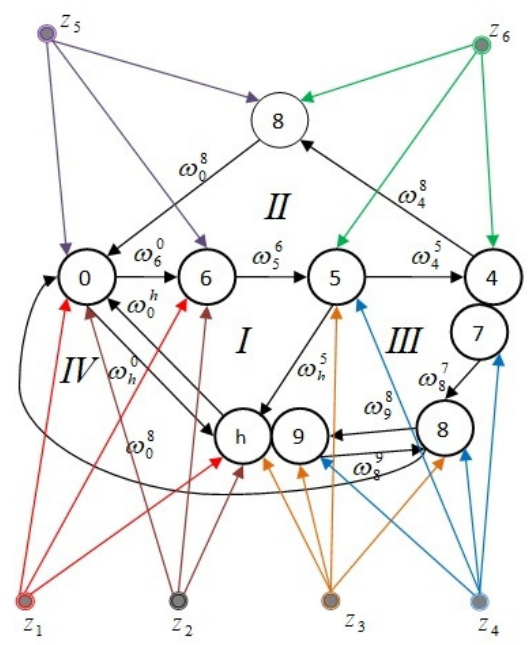

Fig. 5. Implementation of a search graph for a gear.

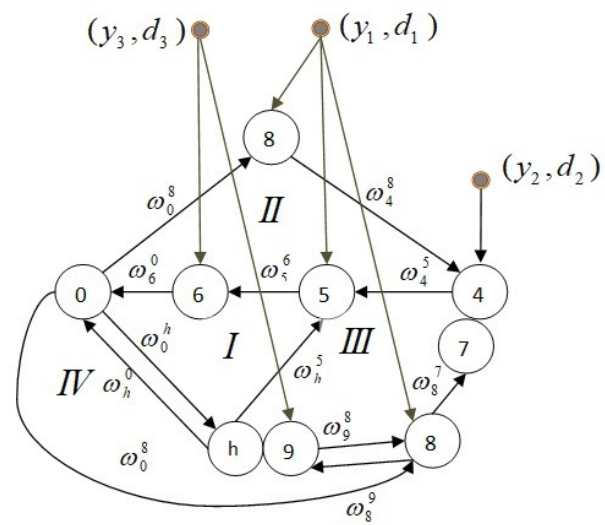

Fig. 6. The signal response for selected graph vertices.

The variables $\left(z_{1}, z_{2}, z_{3}, z_{4}, z_{5}, z_{6}\right)$ in Figure 5 are components of the input vector $Z$. The output of the graph consists of neurons marked by their output signals $\left(y_{1}, y_{2}, y_{3}\right)$ and their corresponding set signals $d_{1}, d_{2}, d_{3}$. When creating an attached $\widehat{G}$ (Figure 6), the output nodes of the normal graph are extracted, which are now available as input nodes for the $\widehat{G}$ graph. Input signal values can now be expressed as $\varepsilon_{1}=y_{1}-d_{1}, \varepsilon_{2}=y_{2}-d_{2}, \varepsilon_{3}=y_{3}-d_{3}$. For searching, the range of values for the number of teeth searched are assumed as: $z_{1}, \quad z_{2}, \quad z_{3}, \quad z_{4}, z_{5}, z_{6}, z_{7}, z_{8}, z_{9}$ : $z_{1} \in(0,1,2, \ldots, 100), z_{2} \in(0,1,2, \ldots, 100), \ldots \ldots z_{9} \in(0,1,2, \ldots, 100)$. After steps $T$, the solution $z_{1}(T), z_{2}(T), z_{3}(T) \ldots$ is obtained, which can be considered as the solution to the search graph. In the case of 
a software solution, a discrete approach is used. In this way, with assumed startup conditions (selection of the initial graph vertex), an iterative string is created that leads to the correct solution. An analytical record of such a string for the initial vertices of graph from Figure 6 can be represented by the analytical expressions (15)-(20), where $G_{i}$ is the iterative search string from the initial vertex $i$.

$$
\begin{aligned}
& G_{Z 7}^{++}=\left({ } ^ { 0 } g _ { 7 } \left({ } ^ { 1 } \omega _ { 8 } ^ { 7 } g _ { 8 _ { a } } \left({ } ^ { 2 } \omega _ { 0 } ^ { 8 } g _ { 0 } { } ^ { 3 } { } ^ { 3 } \omega _ { 6 } ^ { 0 } g _ { 6 } \left({ } ^ { 4 } \omega _ { 5 } ^ { 6 } g _ { 5 } \left({ } ^ { 5 } \omega _ { 4 } ^ { 5 } g _ { 4 } \left({ }^{6} g_{4} g_{7}, \omega_{4}^{8} g_{8 b}\right.\right.\right.\right.\right.\right. \\
& \left.\left.\left.\left.\left.\left.\left.\left({ }^{7} \omega_{0}^{8} g_{0}\right)^{7}\right)^{6}, \omega_{h}^{5} h_{2}\left({ }^{6} \omega_{0}^{h} g_{0}, h_{2} g_{9}\left({ }^{7} \omega_{8}^{9} g_{8_{a}}\right)^{7}\right)^{6}\right)^{5}\right)^{4}, \omega_{h}^{0} h_{2}\right)^{3}\right)^{2}, g_{4} g_{7}\right)^{1}\right)^{0} \\
& G_{Z 6}^{++}=\left(^ { 0 } g _ { 6 } \left({ }^{1} \omega_{5}^{6} g_{5}{ }^{2} \omega_{4}^{5} g_{4}{ }^{3} \omega^{8}{ }_{4}^{8} g_{8_{a}}{ }^{4} \omega_{0}^{8} g_{0}{ }^{5} \omega_{6}^{0} g_{6}, \omega_{h}^{0} h{ }^{6} h_{2} g_{9}\right.\right. \\
& \left.\left.\left.\left.\left.\left.\left(\omega_{8}^{9} g_{8_{b}}\left({ }^{8} \omega_{0}^{8} g_{0}, \omega_{9}^{8} g_{9}\right)^{8}\right)^{7}, \omega_{0}^{h} g_{0}\right)^{6}\right)^{5}\right)^{4}, g_{4} g_{7}\left({ }^{4} \omega_{8}^{7} g_{8_{b}}\right)^{4}\right)^{3}, \omega_{h}^{5} h_{2}\right)^{2}\right)^{1} \\
& G_{Z 5}^{++}=\left({ } ^ { 0 } g _ { 5 } \left({ } ^ { 1 } \omega _ { 4 } ^ { 5 } g _ { 4 } \left({ } ^ { 2 } \omega _ { 4 } ^ { 8 } g _ { 8 _ { a } } { } ^ { 3 } \omega _ { 0 } ^ { 8 } g _ { 0 } \left({ }^{4} \omega_{6}^{0} g_{6}\left({ }^{5} \omega_{5}^{6} g_{5}\right)^{5}, \omega_{h}^{0} h\left({ }^{5} h_{2} g_{9}\right.\right.\right.\right.\right. \\
& \left.\left.\left.\left.\left({ }^{6} \omega_{8}^{9} g_{8_{b}}\left({ }^{7} \omega_{0}^{8} g_{0}, \omega_{9}^{8} g_{9}\right)^{7}\right)^{6}, \omega_{0}^{h} g_{0}\right)^{5}\right)^{4}\right)^{3}, g_{4} g_{7}\left({ }^{3} \omega_{8}^{7} g_{8_{b}}\right)^{3}\right)^{2} \text {, } \\
& \left.\left.\omega_{h}^{5} h_{2}\right)^{1}\right)^{0} \\
& G_{Z 9}^{++}=\left({ } ^ { 0 } g _ { 9 } { } ^ { 1 } \omega _ { 8 } ^ { 9 } g _ { 8 } \left({ } ^ { 2 } \omega _ { 0 } ^ { 8 } g _ { 0 } { } ^ { 3 } { } ^ { 3 } \omega _ { 6 } ^ { 0 } g _ { 6 } \left({ } ^ { 4 } \omega _ { 5 } ^ { 6 } g _ { 5 } { } ^ { 5 } \omega _ { 4 } ^ { 5 } g _ { 4 } \left({ }^{6} g_{4} g_{7}\left({ }^{7} \omega_{8}^{7} g_{8 b}\right)^{7}\right.\right.\right.\right. \text {, } \\
& \left.\left.\left.\left.\left.\left.\omega_{4}^{8} g_{8_{a}}\left(\omega_{0}^{8} g_{0}\right)^{7}\right)^{6}, \omega_{h}^{5} h_{2}\left({ }^{6} h_{2} g_{9}, \omega_{0}^{h} g_{0}\right)^{6}\right)^{5}\right)^{4},\left(\omega_{h}^{0} h_{2}\right)^{3}, \omega_{9}^{8} g_{9}\right)^{2}, h_{2} g_{9}\right)^{1}\right)^{0} \\
& \mathrm{G}_{\mathrm{Z} 8}^{++}=\left({ } ^ { 0 } \mathrm { g } _ { 8 } \left({ } ^ { 1 } \mathrm { w } _ { 0 } ^ { 8 } \mathrm { g } _ { 0 } \left({ } ^ { 2 } \mathrm { w } _ { 6 } ^ { 0 } \mathrm { g } _ { 6 } \left({ } ^ { 3 } \mathrm { w } _ { 5 } ^ { 6 } \mathrm { g } _ { 5 } \left({ } ^ { 4 } \mathrm { w } _ { 4 } ^ { 5 } \mathrm { g } _ { 4 } \left({ }^{5} \mathrm{w}_{4}^{8} \mathrm{~g}_{8_{\mathrm{a}}}, \mathrm{g}_{4} \mathrm{~g}_{7}\left({ }^{6} \mathrm{w}_{8}^{7} \mathrm{~g}_{8_{6}}\right.\right.\right.\right.\right.\right.\right. \\
& \left.\left({ }^{7} \mathrm{w}_{0}^{8} \mathrm{~g}_{0}, \mathrm{w}_{9}^{8} \mathrm{~g}_{9}\left({ }^{8} \mathrm{~h}_{2} \mathrm{~g}_{9}\left({ }^{9} \mathrm{w}_{\mathrm{o}}^{\mathrm{h}} \mathrm{g}_{0}\right)^{8}, \mathrm{w}_{8}^{9} \mathrm{~g}_{8_{\mathrm{b}}}\right)^{7}\right)^{6}\right)^{5}, \\
& \left.\left.\left.\left.\left.\left.\mathrm{w}_{\mathrm{h}}^{5} \mathrm{~h}_{2}\right)^{4}\right)^{3}, \mathrm{w}_{\mathrm{h}}^{0} \mathrm{~h}_{2}\right)^{2}\right)^{1}\right)^{0}\right)^{2} \\
& G_{Z 0}^{++}={ }^{0} g_{0}\left({ } ^ { 1 } \omega _ { 6 } ^ { 0 } g _ { 6 } \left({ } ^ { 2 } \omega _ { 5 } ^ { 6 } g _ { 5 } \left({ } ^ { 3 } \omega _ { 4 } ^ { 5 } g _ { 4 } \left({ }^{4} \omega_{4}^{8} g_{8_{a}}\left({ }^{5} \omega_{0}^{8} g_{0}\right)^{5}, g_{4} g_{7}\left({ }^{5} \omega_{8}^{7} g_{8 b}\right.\right.\right.\right.\right. \\
& \left.\left.\left.\left.\left.\left.\left({ }^{6} \omega_{9}^{8} g_{9}\left({ }^{7} \omega_{8}^{9} g_{8}, g_{9} h_{2}\left({ }^{8} \omega_{0}^{h} g_{0}\right)^{8}\right)^{7}, \omega_{0}^{8} g_{0}\right)^{6}\right)^{5}\right)^{4}, \omega_{h}^{5} h_{2}\right)^{3}\right)^{2}, \omega_{h}^{0} h\right)^{1}\right)^{0}
\end{aligned}
$$

If we have a certain $n$-complex system, then the vertices of the search graph correspond to the teams in $E$ and the set of arcs.

$$
\begin{gathered}
Z \subseteq\left\{z_{1}, z_{2}, \ldots, z_{n-2}\right\} \\
U=\left\{\left(e_{i}, e_{j}\right) \in E \times E /\left(e_{i}, e_{j}\right) \in U \Leftrightarrow u_{i} \text { tests } u_{i} \text { in } Z\right\}
\end{gathered}
$$

Ultimately, the search graph is a function $\widehat{G}=\left(E, U, f_{d}\right)$, where $f_{d}$ determines the optimal number of teeth. For a given gear design, there are groups of three gears with the same number of teeth:

$$
I: z_{1}=z_{4}=z_{7} ; I I: z_{2}=z_{5}=z_{8} ; I I I: z_{3}=z_{6}=z_{9} \text {. }
$$

Figures 7-9 show the examples of the graphical process of finding the optimal number of teeth $z_{1}-z_{9}$ with the help of computer software. The given values are: number of teeth $z_{1}=z_{4}=\mathrm{Z}_{7}=18, z_{2}=z_{5}=\mathrm{Z}_{8}=27$, module value $m=2$, input angular speed (after commissioning) $\omega_{6,0}=377 \mathrm{rad} / \mathrm{s}$, input angular acceleration (during the $8 \mathrm{~s}$ start-up) $\varepsilon_{6,0}=47.1 \mathrm{rad} / \mathrm{s}^{2}$, output angular velocity $\omega_{9,0}=153.2 \mathrm{r} / \mathrm{s}$, and $\varepsilon_{9,0}=19.15 \mathrm{rad} / \mathrm{s}^{2}$.

The analysis and calculations should be carried out for the other DE1, DE2, DE3, DE4 gears and for the reverse gear. In the general notation, the algorithm for generating the optimal number of teeth is presented in Figure 10.

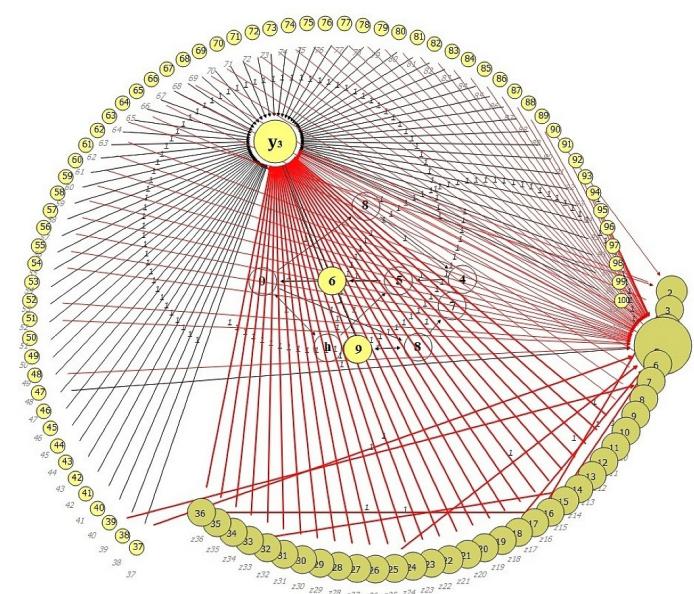

Fig. 7. The process of finding the optimal number for group I. Calculated number of teeth $n=36$.

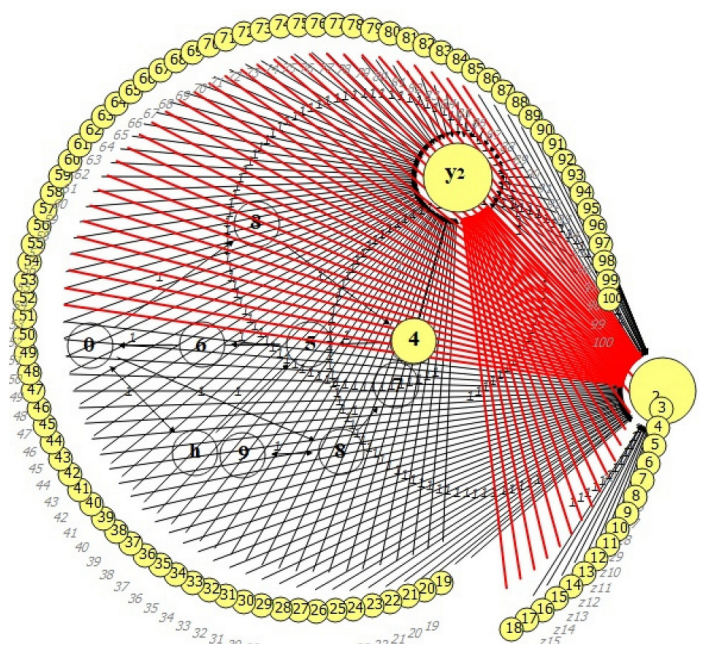

Fig. 8. The process of finding the optimal number for group II. Calculated number of teeth $n=18$.

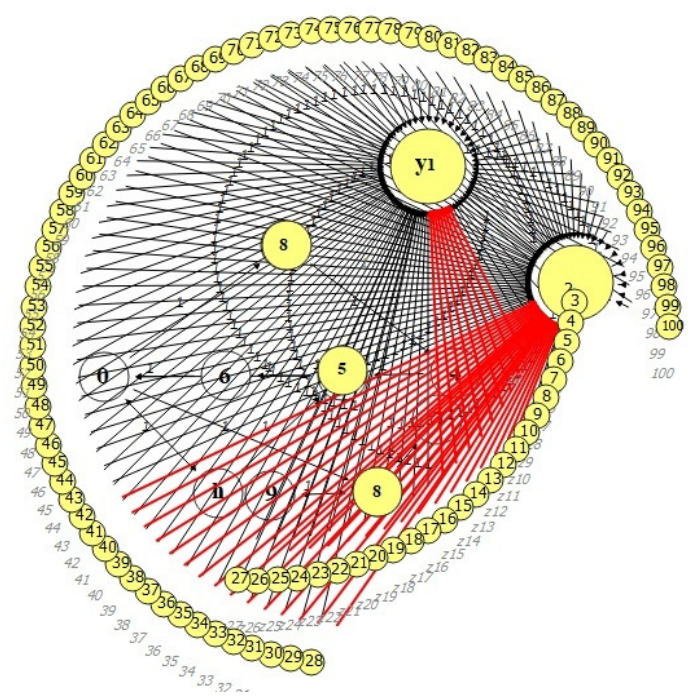

Fig. 9. The process of finding the optimal number for group III Calculated number of teeth $n=27$. 


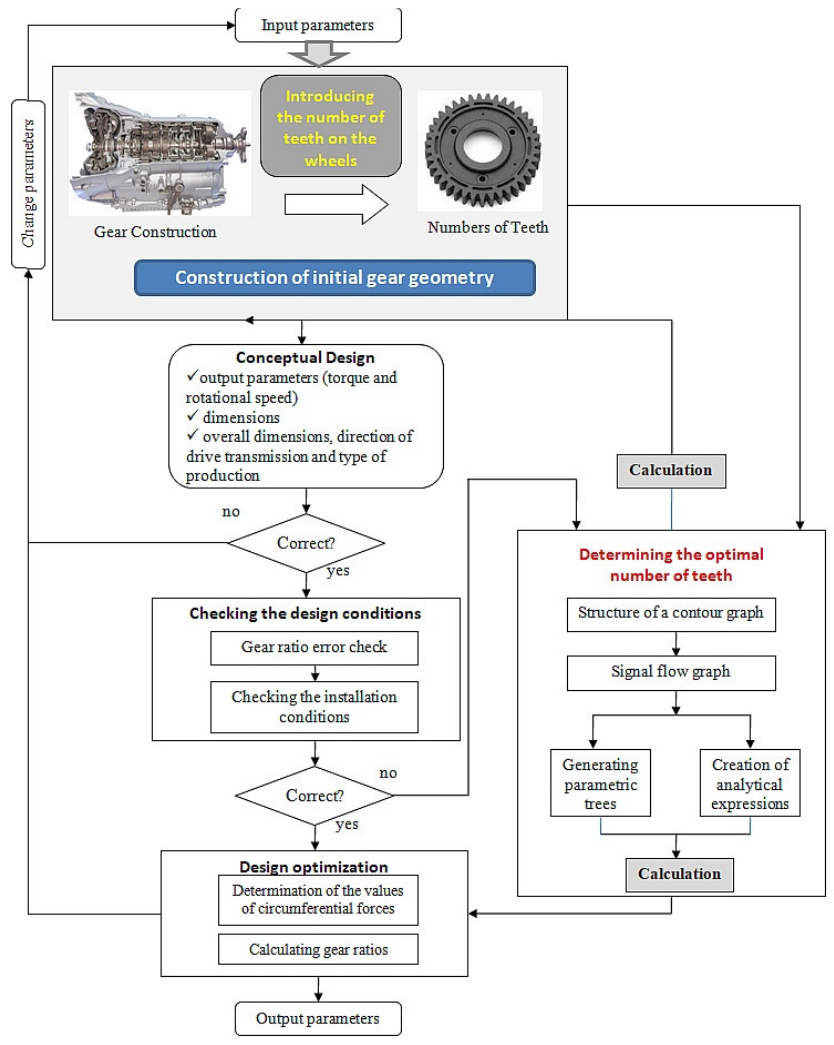

Fig. 10. Flowchart of the general optimization algorithm of a transmission system with selection of the appropriate number of teeth.

\section{CONCLUSION}

The graph-based methods of analysis and synthesis of planetary gears provide an alternative for the accomplishing of the tasks in question. The automatic transmission presented in this article was modeled with the use of signal flow graphs. Due to the use of the new modeling method, there is a need to analyze the technical risk of such a solution. An element necessary in the risk assessment process is to take into account the requirements of the engineering design methodology. In this regard, it is worth choosing a design methodology that meets the following criteria:

\section{- Completeness of technical criteria.}

- Adequacy of the set of parameters describing the designed object.

The criteria formulated for assessment should be synthetic because this increases the objectivity of assessment of a given solution. Designing facilities involves increasing efficiency (to some extent) while reducing the efficiency of another team. Determining effectiveness consists in comparing the effects of a given measure with its expenditure. Particularly in this respect, it should be ensured that the risk of the adopted boundary standards in the scope of e.g. costs is not exceeded. The current article presents the application of dependency graphs to determine the optimal number of gear teeth for an automatic transmission. Each gearbox (automatic or manual planetary gear) can be additionally modeled with Hsu graphs [36].

\section{REFERENCES}

[1] K. S. Belkhir, "Intelligent Maximum Power Tracking Control For a Wind Energy System Based on Magnetic Gear Generator," Engineering, Technology \& Applied Science Research, vol. 9, no. 4, pp. 4329-4333, Aug. 2019, https://doi.org/10.48084/etasr.2851.

[2] F. Buchsbaum and F. Freudenstein, "Synthesis of kinematic structure of geared kinematic chains and other mechanisms," Journal of Mechanisms, vol. 5, no. 3, pp. 357-392, Sep. 1970, https://doi.org/10. 1016/0022-2569(70)90068-6.

[3] W. J. Sohn and F. Freudenstein, "An Application of Dual Graphs to the Automatic Generation of the Kinematic Structures of Mechanisms," Journal of Mechanisms, Transmissions, and Automation in Design, vol. 108, no. 3, pp. 392-398, Sep. 1986, https://doi.org/10.1115/1.3258745.

[4] F. R. E. Crossley, "The Permutations of Kinematic Chains of Eight Member or Less from the Graph-Theoretic Viewpoint," Developments in Theoretical and Applied Mechanisms, vol. 2, pp. 467-486, 1965.

[5] F. Freudenstein, "An Application of Boolean Algebra to the Motion of Epicyclic Drives," Journal of Engineering for Industry, vol. 93, no. 1, pp. 176-182, Feb. 1971, https://doi.org/10.1115/1.3427871.

[6] A. Deptuła and M. A. Partyka, "Application of dependence graphs and game trees for decision decomposition for machine systems," Journal of Automation Mobile Robotics and Intelligent Systems, vol. 5, no. 4, pp. 17-26, 2011.

[7] C. A. Nelson and R. J. Cipra, "Simplified Kinematic Analysis of Bevel Epicyclic Gear Trains With Application to Power-Flow and Efficiency Analyses," Journal of Mechanical Design, vol. 127, no. 2, pp. 278-286, Mar. 2005, https://doi.org/10.1115/1.1814392.

[8] X. Yu, J. Zhang, C. Fan, and S. Chen, "Stability analysis of governorturbine-hydraulic system by state space method and graph theory," Energy, vol. 114, pp. 613-622, Nov. 2016, https://doi.org/10.1016/ j.energy.2016.07.164.

[9] N. F. Timerbaev, A. R. Sadrtdinov, and R. G. Safin, "Software Systems Application for Shafts Strength Analysis in Mechanical Engineering," Procedia Engineering, vol. 206, pp. 1376-1381, Jan. 2017, https://doi.org/10.1016/j.proeng.2017.10.648.

[10] J. Xu et al., "The Construction of Design Science Knowledge Graphs Based on National Natural Science Foundation of China," Proceedings of the Design Society: International Conference on Engineering Design, vol. 1, no. 1, pp. 2607-2616, Jul. 2019, https://doi.org/10.1017/ dsi.2019.267.

[11] G. Chatterjee and L.-W. Tsai, "Enumeration of Epicyclic-Type Automatic Transmission Gear Trains," SAE Transactions, vol. 103, pp. 1415-1426, 1994.

[12] G. Gururaj and K. S. Gowri, "Performance Optimization of an eCAR by Parametric Analysis," Engineering, Technology \& Applied Science Research, vol. 9, no. 6, pp. 4968-4973, Dec. 2019, https://doi.org/ 10.48084/etasr.3139.

[13] A. Y. Al-Rawashdeh, "Investigation of an Induction Wound Rotor Motor to Work as a Synchronous Generator," Engineering, Technology \& Applied Science Research, vol. 9, no. 2, pp. 4071-4074, Apr. 2019, https://doi.org/10.48084/etasr.2606.

[14] G. Kron, "Generalized Theory of Electrical Machinery," Transactions of the American Institute of Electrical Engineers, vol. 49, no. 2, pp. 666683, Apr. 1930, https://doi.org/10.1109/T-AIEE.1930.5055554.

[15] J. Wojnarowski, "The graph method of determining the loads in complex gear trains," Mechanism and Machine Theory, vol. 11, no. 2, pp. 103121, Jan. 1976, https://doi.org/10.1016/0094-114X(76)90003-3.

[16] D. J. Sanger, "The determination of power flow in multiple-path transmission systems," Mechanism and Machine Theory, vol. 7, no. 1, pp. 103-109, Mar. 1972, https://doi.org/10.1016/0094-114X(72)900201 .

[17] F. Freudenstein and L. Dobrjanskyj, "On a theory for the type synthesis of mechanisms," in Applied Mechanics, H. Gortler, Ed. Berlin, Heidelberg: Springer, 1966, pp. 420-428.

[18] L.-W. Tsai and C.-C. Lin, "The Creation of Nonfractionated, TwoDegree-of-Freedom Epicyclic Gear Trains," Journal of Mechanisms, 
Transmissions, and Automation in Design, vol. 111, no. 4, pp. 524-529, Dec. 1989, https://doi.org/10.1115/1.3259033.

[19] C. - H. Hsu, "Graph notation and kinematic equations of motion of planetary differential gear trains," International Journal of Vehicle Design, vol. 13, no. 3, pp. 233-241, Jan. 1992, https://doi.org/ 10.1504/IJVD.1992.061725.

[20] H.-S. Yan and Y.-T. Chiu, "An algorithm for the construction of generalized kinematic chains," Mechanism and Machine Theory, vol. 62, pp. 75-98, Apr. 2013, https://doi.org/10.1016/j.mechmachtheory. 2012.11.005.

[21] D. B. Marghitu, Kinematic Chains and Machine Components Design, 1st edition. Amsterdam, Boston: Academic Press, 2005.

[22] A. Deptuła, J. Drewniak, and M. A. Partyka, "Application of Dependence Graphs and Game Trees in Analysis of a Planetary Gear Modeled With a Contour Graph," Machine Dynamics Research, vol. 41, no. 3, pp. 105-114, 2017.

[23] D. Gibson and S. Kramer, "Symbolic Notation and Kinematic Equations of Motion of the Twenty-Two Basic Spur Planetary Gear Trains," Journal of Mechanisms, Transmissions, and Automation in Design, vol. 106, no. 3, pp. 333-340, Sep. 1984, https://doi.org/10.1115/1.3267416.

[24] J. M. del Castillo, "Enumeration of 1-DOF Planetary Gear Train Graphs Based on Functional Constraints," Journal of Mechanical Design, vol. 124, no. 4, pp. 723-732, Nov. 2002, https://doi.org/10.1115/1.1514663.

[25] A. C. Rao and P. B. Deshmukh, "Computer aided structural synthesis of planar kinematic chains obviating the test for isomorphism," Mechanism and Machine Theory, vol. 36, no. 4, pp. 489-506, Apr. 2001, https://doi.org/10.1016/S0094-114X(00)00030-6.

[26] H. Ding, Z. Huang, and Y. Cao, "The topological graphs creation automatically of kinematic chains and atlas database establishment," Journal of Mechanical Engineering, vol. 42, no. 4, pp. 32-36, 2006.

[27] J. Wojnarowski, J. Kopec, and S. Zawislak, "Gears and graphs," Journal of Theoretical and Applied Mechanics, vol. 44, no. 1, pp. 139-162, 2006.

[28] M.-C. Tsai, C.-C. Huang, and B.-J. Lin, "Kinematic Analysis of Planetary Gear Systems Using Block Diagrams," Journal of Mechanical Design, vol. 132, May 2010, Art. no. 065001, https://doi.org/ $10.1115 / 1.4001598$.

[29] J. Drewniak, S. Zawislak, and A. Wieczorek, "Analysis method of automatic planetary transmission kinematics," Scientific Journal of Silesian University of Technology-Series Transport, vol. 83, pp. 65-71, 2014.

[30] A. Deptula, J. Drewniak, and M. A. Partyka, "Selection of the Optimal Number of Teeth for a Biplanetary Bevel Gear Based on Discrete Optimization," Machine Dynamics Research, vol. 42, no. 3, pp. 55-66, 2018.

[31] L. P. Laus, H. Simas, and D. Martins, "Efficiency of gear trains determined using graph and screw theories," Mechanism and Machine Theory, vol. 52, pp. 296-325, Jun. 2012, https://doi.org/10.1016/ j.mechmachtheory.2012.01.011.

[32] J. R. Goma Ayats, U. Diego-Ayala, J. Minguella Canela, F. Fenollosa, and J. Vivancos, "Hypergraphs for the analysis of complex mechanisms comprising planetary gear trains and other variable or fixed transmissions," Mechanism and Machine Theory, vol. 51, pp. 217-229, May 2012, https://doi.org/10.1016/j.mechmachtheory.2011.07.011.

[33] W. Yang, H. Ding, B. Zi, and D. Zhang, "New Graph Representation for Planetary Gear Trains," Journal of Mechanical Design, vol. 140, Nov. 2017, Art. no. 012303, https://doi.org/10.1115/1.4038303.

[34] A. Deptula, Application of graphic decision structures in the design and management methodology. Opole, Poland: Publishing House of the Opole University of Technology, 2020.

[35] M. S. Pishvaee and M. Rabbani, "A graph theoretic-based heuristic algorithm for responsive supply chain network design with direct and indirect shipment," Advances in Engineering Software, vol. 42, no. 3, pp. 57-63, Mar. 2011, https://doi.org/10.1016/j.advengsoft.2010.11.001.

[36] C.-H. Hsu, "An Analytic Methodology for the Kinematic Synthesis of Epicyclic Gear Mechanisms," Journal of Mechanical Design, vol. 124, no. 3, pp. 574-576, Aug. 2002, https://doi.org/10.1115/1.1485094. 